This item was submitted to Loughborough's Research Repository by the author.

Items in Figshare are protected by copyright, with all rights reserved, unless otherwise indicated.

\title{
Effect of virtual reality and whole-body heating on motion sickness severity: A combined and individual stressors approach
}

PLEASE CITE THE PUBLISHED VERSION

\section{PUBLISHER}

Elsevier B.V.

\section{VERSION}

AM (Accepted Manuscript)

\section{PUBLISHER STATEMENT}

This paper was accepted for publication in the journal Displays and the definitive published version is available at https://doi.org/10.1016/j.displa.2019.08.007.

\section{LICENCE}

CC BY-NC-ND 4.0

\section{REPOSITORY RECORD}

Arnold, Josh, Kate O'Keeffe, Chloe McDaniel, Simon Hodder, and Alexander Lloyd. 2019. "Effect of Virtual Reality and Whole-body Heating on Motion Sickness Severity: A Combined and Individual Stressors Approach". Loughborough University. https://hdl.handle.net/2134/9741200.v1. 
1 TITLE: Effect of virtual reality and whole-body heating on motion sickness severity: A

AUTHORS: Josh T. Arnold (MRes.) ${ }^{1}$, Kate O’Keeffe (MSc.) ${ }^{1}$, Chloe McDaniel (MSc.) ${ }^{1}$,

AFFILIATIONS: ${ }^{1}$ Environmental Ergonomics Research Centre, Loughborough University, Leicestershire, UK.

RUNNING HEAD: Motion sickness in heat and virtual reality.

MANUSCRIPT TYPE: Research Article

WORD COUNT: 3485

CORRESPONDENCE: Alex Lloyd, PhD. Environmental Ergonomics Research Centre,

A.1loyd@lboro.ac.uk

ACKNOWLEDGEMENTS: G.A Raccuglia is acknowledged for his help and support

21 during data collection.

CONFLICTS OF INTEREST AND SOURCES OF FUNDING: The authors declare they

24 have no conflict of interest and the study did not receive funding from external sources to

25 Loughborough University. 
ABSTRACT

Background: Virtual reality (VR) use is limited by the potential side effects of prolonged exposure to vection, leading to motion sickness. Air temperature $\left(\mathrm{T}_{\mathrm{a}}\right)$ may exacerbate the severity of such side effects through a synergistic interaction. This study assessed the individual and combined impact of a hot $\mathrm{T}_{\mathrm{a}}$ and VR on motion sickness severity. Method: Thirteen healthy volunteers were exposed to a 20 min visual stimulus, across four experimental conditions: $\mathrm{N}_{-} \mathrm{CS}: 22^{\circ} \mathrm{C} \mathrm{T}_{\mathrm{a}}$ with computer screen; $\mathrm{N} \_$VR: $22^{\circ} \mathrm{C} \mathrm{T}_{\mathrm{a}}$ with VR; H_CS: $35^{\circ} \mathrm{C} \mathrm{T}_{\mathrm{a}}$ with computer screen; H_VR: $35^{\circ} \mathrm{C} \mathrm{T}_{\mathrm{a}}$ with VR. Motion sickness was assessed via fast motion sickness scale (FMS) and simulator sickness questionnaire (SSQ). Physiological indices of motion sickness including, sweat rate, rectal temperature, cutaneous vascular conductance (CVC), skin temperature, blood pressure and heart rate were also examined. Results: FMS and SSQ ratings indicate a significant main effect for VR, increasing sickness severity $(\mathrm{p}<0.001)$. A significant main effect of $\mathrm{T}_{\mathrm{a}}$ was observed for SSQ, but not FMS ratings (FMS, p=0.07; SSQ, $\mathrm{p}<0.04)$. Despite trends towards synergism, no interaction $\left(\mathrm{T}_{\mathrm{a}} \times \mathrm{VR}\right)$ was observed for FMS $(\mathrm{p}=0.2)$ or SSQ scores $(\mathrm{p}=0.07)$, indicating an additive response. Synergistic trends were also observed for sweat rate and CVC.

Conclusion: Synergism between VR and heat on motion sickness remains unclear, possibly as a result of considerable inter-individual variation in the reported subjective responses. Understanding of the questions raised by this study inform safe working guidelines for the use of VR in commercial and occupational settings.

\section{KEY WORDS}




\subsection{INTRODUCTION}

Advances in Virtual Reality technology (VR) has attracted interest across a range of occupational fields, including medical, military and educational industries. For example, users are now able to artificially experience complex occupational scenarios with reduced risk of injury, damage or cost. Yet, despite the efforts of many manufacturers, there are concerns regarding the associated side effects of prolonged VR use, including malaise, dizziness, headache and eyestrain [1,2]. In many cases VR use has shown to increase the risk of visually-induced motion sickness (VIMS), also known as cybersickness, with ongoing debate concerning to the direct influence of perceived self-motion (vection) [3-9]. For example, an observational study comparing ratings of VIMS in 497 healthy adults, reported a 55\% vs. $14 \%$ prevalence of sickness, having viewed a 3D and 2D movie respectively [10]. Symptom intensity was also observed to be significantly higher following 3D compared to 2D viewing. Comparable to 'classical' motion sickness, common symptoms of VIMS may present as dizziness, vertigo, sweating, stomach awareness and nausea, which may further progress to vomiting with sufficient stimulus duration or intensity [11-13]. Given the scope and utility of VR technology, it is important to better understand the underlying mechanisms, mediating factors and interactions which may link VR with VIMS, thus maximising its future potential.

Due to the complex nature of motion sickness there is limited agreement on a holistic and theoretical understanding of the mechanisms that cause the syndrome [14]. Indeed, it has even been reported that motion sickness can develop prior to exposure to a provocative stimulus, perhaps due to expectancy or anxiety effects [14]. At present, several hypotheses exist to explain the cause of motion sickness; (i) Sensory Conflict and Rearrangement Theory [15], in which sensory information from visual, vestibular and somatosensory systems, either become mismatched, or if these senses fail to match those stored in the central nervous system from 
past experiences; (ii) Postural Instability Theory [16], in which an organism attempts to maintain postural stability in relation to its environment throughout daily activities, with sickness occurring when a stable state can no longer be obtained, along with a perceived lack of control; (iii) Poison Theory or the Toxic Hypothesis [17], an evolutionary response in which emesis acts as a defence mechanism to intoxication of the body due to toxin induced stimulation of the vestibular senses. The net result of the latter is a mismatch between perceived moving vestibular and static visual signals, consequently leading to emesis, vertigo, dizziness and postural instability.

There is also limited research on the secondary and mediating factors that subsequently impact upon VIMS susceptibility during VR use. One such factor is air temperature $\left(T_{a}\right)$, including both hot and cold stimuli. Interestingly, previous studies have highlighted interactions between motion sickness and thermoregulation, showing an increased risk of deep-body cooling in motion sick individuals, when exposed to cold environments $[18,19]$. In view of the toxic hypothesis, its appears conceivable that this reduction in deep-body temperature may act in conjunction with motion sickness, to further protect the body against perceived intoxication via a slowing of metabolic rate [20]. We further postulate that an added thermoregulatory load induced via heat stress, in addition to a provocative VR stimulus, may artificially strengthen the body's belief that it is under threat from intoxication, subsequently triggering a heightened nauseogenic response [20]. As such, it is possible that a mechanistic interaction of a synergistic nature may exist between heat exposure (i.e. prolonged exposures to $\mathrm{T}_{\mathrm{a}}>30^{\circ} \mathrm{C}$ ) and VR use, on VIMS susceptibility [21]. While the toxic hypothesis provides a potential explanation for a synergistic interaction between heat and VR, if an interaction is not observed (i.e. additive effects), this may better support the role of other theories such as sensory conflict, in the aetiology of motion sickness. 
102 To understand the role of a hot air temperature in modulating nausea in virtual reality, this

103 investigation examined the combined and differential impacts of heat and VR on motion

104 sickness severity. Three hypotheses were constructed; 1 . individuals would report

105 significantly greater perceptions of VIMS whilst viewing VR, when compared to a computer

106 screen control, 2. individuals would not report any difference in VIMS under hot conditions,

107 compared to a thermoneutral control, 3. combined VR and hot conditions would

108 synergistically interact to significantly increase VIMS ratings when compared to either factor

109 independently. An understanding of such main effects and potential interactions is likely to

110 better inform safe working guidelines for the use of VR in commercial and occupational

111 settings, as well as elucidating some of the underlying mechanisms impacting VIMS.

\subsection{METHODS}

\subsection{Participants}

115 Thirteen healthy volunteers, five male and eight females (age, $25 \pm 3$ yrs), were recruited

116 from the Loughborough, UK between June and November 2017. Inclusion criteria detailed:

117 non-smoking, otherwise healthy individuals, reporting no significant ( $>6 \mathrm{hrs}$ per week)

118 gaming or VR experience. All participants provided written informed consent. Ethical

119 approval was granted by the Loughborough University Ethics Committee and the research

120 was conducted in accordance with the Declaration of Helsinki, 2008.

\subsection{Study Design}

123 The study utilised a repeated measures randomised design and was conducted in the

124 Environmental Ergonomics Research Centre at Loughborough University. Participants were

125 exposed to a standardised visual stimulus, presented across four independent experimental 
conditions in a random order: N_CS: neutral air temperature with computer screen $\left(\mathrm{T}_{\mathrm{a}}, 22.2 \pm\right.$ $1.0{ }^{\circ} \mathrm{C}$; RH, $\left.40 \pm 3 \%\right)$; N_VR: neutral air temperature with VR headset $\left(\mathrm{T}_{\mathrm{a}}, 22.3 \pm 1.0{ }^{\circ} \mathrm{C}\right.$; RH, $40 \pm 4 \%)$; H_CS: hot air temperature with computer screen $\left(\mathrm{T}_{\mathrm{a}}, 35.0 \pm 0.9{ }^{\circ} \mathrm{C} ; \mathrm{RH}, 38 \pm\right.$ $3 \%)$; H_VR: hot air temperature maintained with VR headset $\left(\mathrm{T}_{\mathrm{a}}, 35.1 \pm 0.9^{\circ} \mathrm{C} ; \mathrm{RH}, 37 \pm 4\right.$ $\%)$. One week prior to the first trial, participants were fully briefed in relation to the study aim, design and test requirements. Twenty-four hours prior to each laboratory visit, participants were asked to refrain from alcohol, caffeine and gaming use, and avoid heat exposure. Participants commenced all tests at the same time each day to reduce the effect of circadian rhythm. A minimum $24 \mathrm{hr}$ wash-out period was observed between trials to eliminate any carry-over of effects. Air temperature and relative humidity were monitored throughout the investigation via Testo probe and data logger (Probe- 0635 1535, Logger- 435, Testo Ltd, Germany), and maintained within $1.0{ }^{\circ} \mathrm{C} \mathrm{T}_{\mathrm{a}} / 5 \% \mathrm{RH}$ of the desired set-point for each trial.

\subsection{Visual Stimulus.}

140 The visual stimulus consisted of a 20 min pre-recorded series of computer-generated driving

141 clips (5 merged clips at 3 to 5 mins in duration each), presented through software Project

142 Cars $^{\mathrm{TM}}$ (Slightlymad Studious Ltd.). In the control conditions, participants observed the clip

143 sequence on a computer screen (HP LA2306x, 23", $1920 \times 1080)$ positioned $1 \mathrm{~m}$ away at

144 head height. The same driving clip was presented to participants using a VR headset (Oculus

145 Rift Developmental Kit 2), offering a $100^{\circ}$ horizontal field of view, with 960x1080 resolution 146 in each eye.

148 Pre-recorded videos were used to potentiate motion sickness, due to a reduction in sense of 149 control that the participant experiences relative to their environment [22]. Furthermore, the 150 sequencing of the clips was strategically ordered based on the number of turns in the track 
151 (least to most), with the assumption that more turns would equate to an increased risk of

152 motion sickness [23]. Each video clip was presented from the perspective of the driver. Whilst

153 computer screen conditions were fixed in 2D, VR conditions allowed participants to

154 manipulate their visual array in accordance with the pitch, roll and yaw of their own head.

155 Fixation was not controlled in the current study to better align with real world scenarios.

156 Sound levels were standardised across all conditions using a commercial 2.1 multimedia

157 speaker system (Phillips Ltd., Netherlands).

158

159

\subsection{Experimental Procedures}

160 On arrival to the laboratory, participants first completed a simulator sickness questionnaire

161 (SSQ) to ensure no carry-over of motion sickness symptoms from previous trials [24].

162 Perceptual ratings for 16 motion sickness symptoms were provided on a 4-point scale (0-

163 none, 1-slight, 2-moderate, 3-severe), and added together to provide a total score. In a

164 separate preparation room, participants donned the appropriate experimental apparatus; heart

165 rate assessed by 3-lead electrocardiogram (Tango M2, SunTech Medical Inc., USA), skin

166 temperature at the calf, thigh, pectoral and tricep by surface thermistor (iButton ${ }^{\mathrm{TM}}$, Maxim,

167 USA), deep-body temperature by rectal thermistor (Grant Instruments Ltd., UK), local sweat

168 rate at the palm, mid-upper-back and shin by ventilated sweat capsules (Q-Sweat, TestWorks,

169 WR Medical Co., USA), skin blood flow at the inner forearm by laser doppler (Moor

170 Instruments Ltd, Devon) and blood pressure by automated sphygmomanometer (Tango M2,

171 SunTech Medical Inc., USA). Once ready, participants entered the temperature controlled

172 experimental room, were asked to sit in front of the computer system on a car seat and find a

173 comfortable position which could be maintained with minimal need for movement.

174 
175 Participants undertook a 5-min acclimatisation period, remaining quiet and still. At this point,

176 all equipment was calibrated and zeroed ready for data collection. Participants were also

177 briefed on the subsequent use of the Fast Motion Sickness Scale (FMS), used to

178 instantaneously assess perceived severity of motion sickness. The FMS was presented via a

179 visual-analogue scale ranging 0 (no sickness) to 20 (incapacitating sickness), in which a score

180 of 10 should represent moderate levels of motion sickness. The use of this simplistic scale

181 allowed for easy memorisation and recall during VR conditions in which the headset was

182 used. Participants were asked to focus on nausea, general discomfort and stomach awareness,

183 and to ignore perceptions such as boredom, fatigue and nervousness [25]. At minute five, a

184 member of the research team initiated the 20-min visual stimulus, either via computer screen

185 or VR headset. After each minute, participants were asked to provide a verbal FMS rating.

186 Heart rate, skin temperature, rectal temperature, local sweat rate and skin blood flow were all

187 continuously assessed throughout the 20-min period, sampled at $1 \mathrm{~Hz}$. Blood pressure, was

188 assessed every 5 mins. On completion of the 20-min visual stimulus, the SSQ were

189 immediately completed once again, after which the trial finished. To complete the

190 experiment, participants were handed a last copy of the SSQ when leaving the laboratory in

191 order to record simulator sickness two hours post-visual stimulus.

192

\section{$193 \quad 2.5$ Data Analysis}

194 Statistical significance was set at $P \leq 0.05$. Two-way, repeated measures analysis of variance

195 (ANOVA) was used to evaluate the main effects of air temperature and visual stimulus,

196 addressing hypothesis 1 and 2 , in addition to the interaction between the two factors $\left(\mathrm{T}_{\mathrm{a}} \mathrm{X}\right.$

197 VR), addressing hypothesis 3. Inferential statistical analysis was conducted using the software

198 package IBM SPSS Statistics for Windows (version 23, IBM Corp., USA). Physiological

199 variables were interpreted as a mean and peak value across each 20 min trial. Data are 
presented as mean and [95\% Confidence Intervals (CI)] unless stated otherwise in figures and 201 tables.

\subsection{RESULTS}

All participants successfully completed the experiment, undertaking all trials, despite varying reports of motion sickness.

\subsection{Motion Sickness}

Taken independently (hypothesis 1), VR significantly increased motion sickness severity compared to computer screen viewing when expressed as mean FMS rating (CS trials, 0.6 [0.2 - 1.1] vs. VR trials, 4.3 [2.6 - 6.0]; $\mathrm{p}<0.001)$. Air temperature, independently of VR

211 (hypothesis 2), did not significantly influence mean FMS ratings ( $N$ trials, 2.1 [1.3 - 2.9] vs. H trials, 2.8 [1.5 - 4.2]; $\mathrm{p}=0.07$ ). No interaction (hypothesis 3) was observed between VR and air temperature $(\mathrm{p}=0.2)$, indicating an additive effect when these factors are combined. Peak

214 FMS ratings reflect the results seen for mean FMS ratings, with a significant main effect 215 observed for VR (CS trials, 1.6 [0.8 - 2.4] vs. VR trials, 8.7 [5.5 - 11.8]; $\mathrm{p}<0.001)$, however no effect for air temperature ( $N$ trials, 4.8 [3.2 - 6.4] vs. $H$ trials, 5.5 [3.1 - 7.9]; p=0.3), and no interaction seen between VR and air temperature $(\mathrm{p}=0.9)$. Whilst statistical analysis of the data reveals no significant evidence for synergism $\left(T_{a} \times V R\right)$, a difference in the magnitude of mean change between H_CS and H_VR $(\Delta$ FMS, 4.0$)$, compared to N_CS and N_VR $(\Delta$

220 FMS, 3.2), indicates a trend towards a synergistic interaction (Fig 1.). Furthermore, inspection of individual data shows a large inter-individual difference in the magnitude of response across subjects, providing partial evidence for distinct groups of responders or nonresponders. Approximately six individuals showed a clear visible difference in mean FMS 
224 rating between control and VR conditions, four individuals showing no visible difference, and

225 three interspersed between (Fig 2.).

226

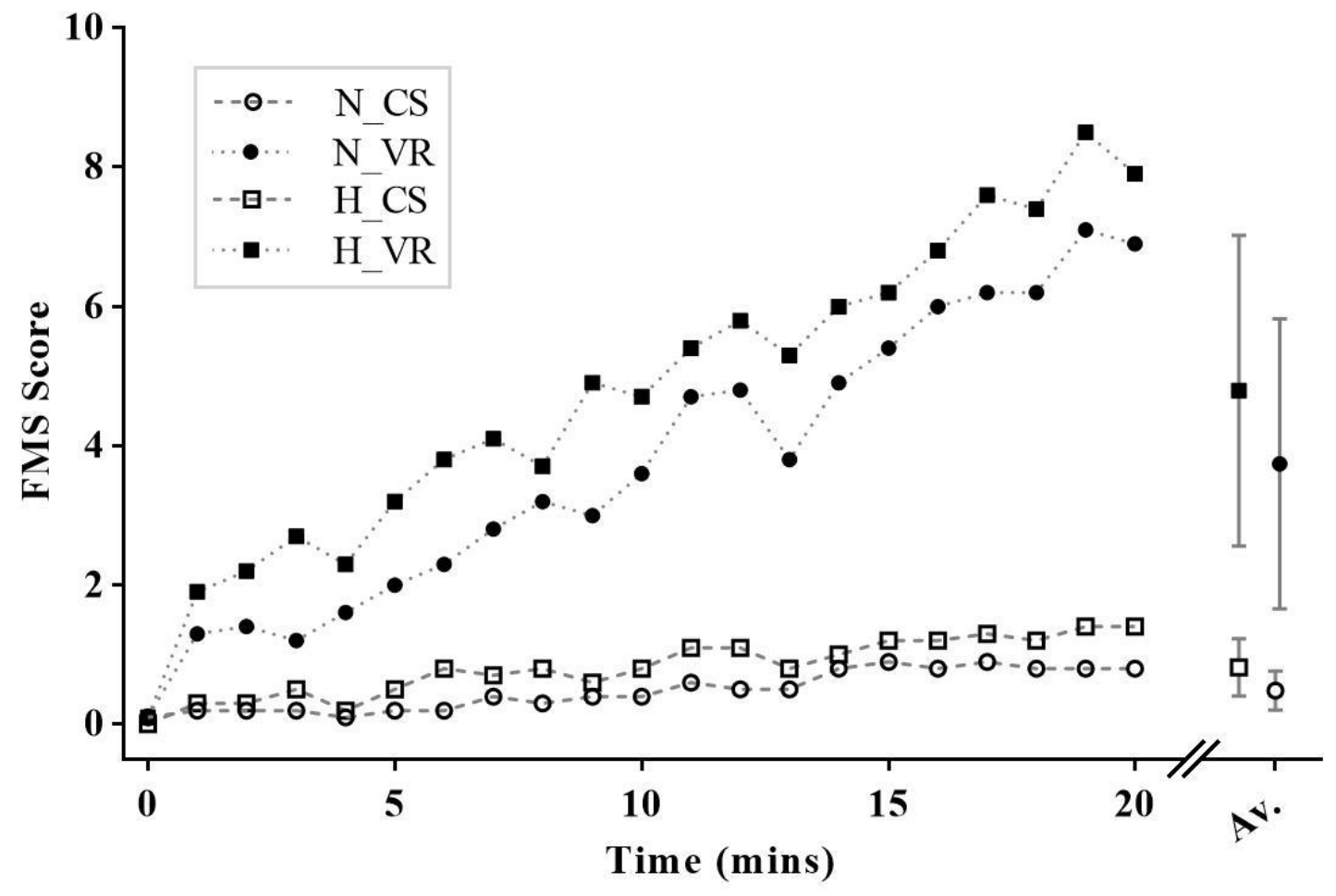

Figure 1: Impact of virtual reality use and air temperature on perceptions of visually induced motion sickness assessed via a Fast Motion Sickness scale.

Note: Data are mean $\pm \mathrm{SD} ; \mathrm{n}=13$. Experimental conditions; N_CS, $22^{\circ} \mathrm{C}$ Ta with computer screen; N_VR, $22^{\circ} \mathrm{C}$ Ta with virtual reality headset; H_CS, $35^{\circ} \mathrm{C}$ Ta with computer screen; H_VR, $35^{\circ} \mathrm{C}$ Ta with virtual reality headset. 


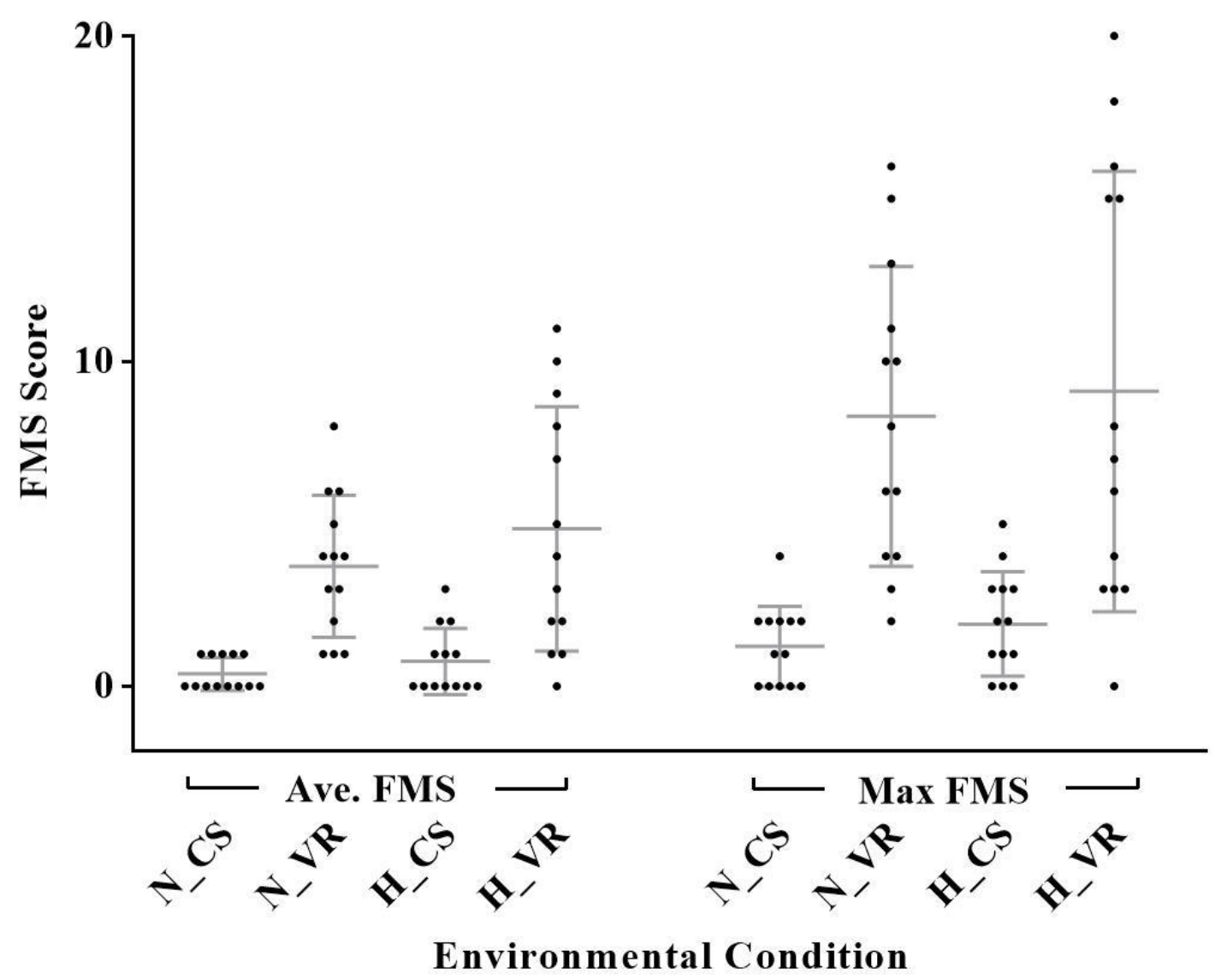

Figure 2: Impact of virtual reality use and air temperature on perceptions of visually induced motion sickness assessed via a Fast Motion Sickness scale.

Note: Data are mean $\pm \mathrm{SD}$, with individual data points presented; $\mathrm{n}=13$. Experimental conditions; N_CS, $22^{\circ} \mathrm{C}$ Ta with computer screen; N_VR, $22^{\circ} \mathrm{C}$ Ta with virtual reality headset; H_CS, $35^{\circ} \mathrm{C}$ Ta with computer screen; H_VR, $35^{\circ} \mathrm{C}$ Ta with virtual reality headset.

230 On arrival to the laboratory, all participants reported zero ratings of motion sickness via SSQ

231 across all trials. The SSQ ratings immediately following each trial paralleled FMS

232 observations. The results demonstrate significant independent main effects (hypothesis 1 and

233 2) for VR (CS trials, 4.1 [1.8 - 6.5] vs. VR trials, 12.8 [6.7 - 19.0]; $\mathrm{p}=0.001)$ and air

234 temperature $(N$ trials, $7.1[3.6-10.6]$ vs. $H$ trials, 9.9 [4.8 - 15.0]; $\mathrm{p}=0.04)$ on reported 
235 motion sickness severity. Again, no significant interaction (hypothesis 3) was observed

236 between VR and air temperature $(\mathrm{p}=0.07)$, although a clear trend for synergism was observed.

237 Perceptions of motion sickness diminished across all conditions at $2 \mathrm{hrs}$ post trial (Fig 3.),

238 with no main effects observed $(\mathrm{p}>0.1)$.

239

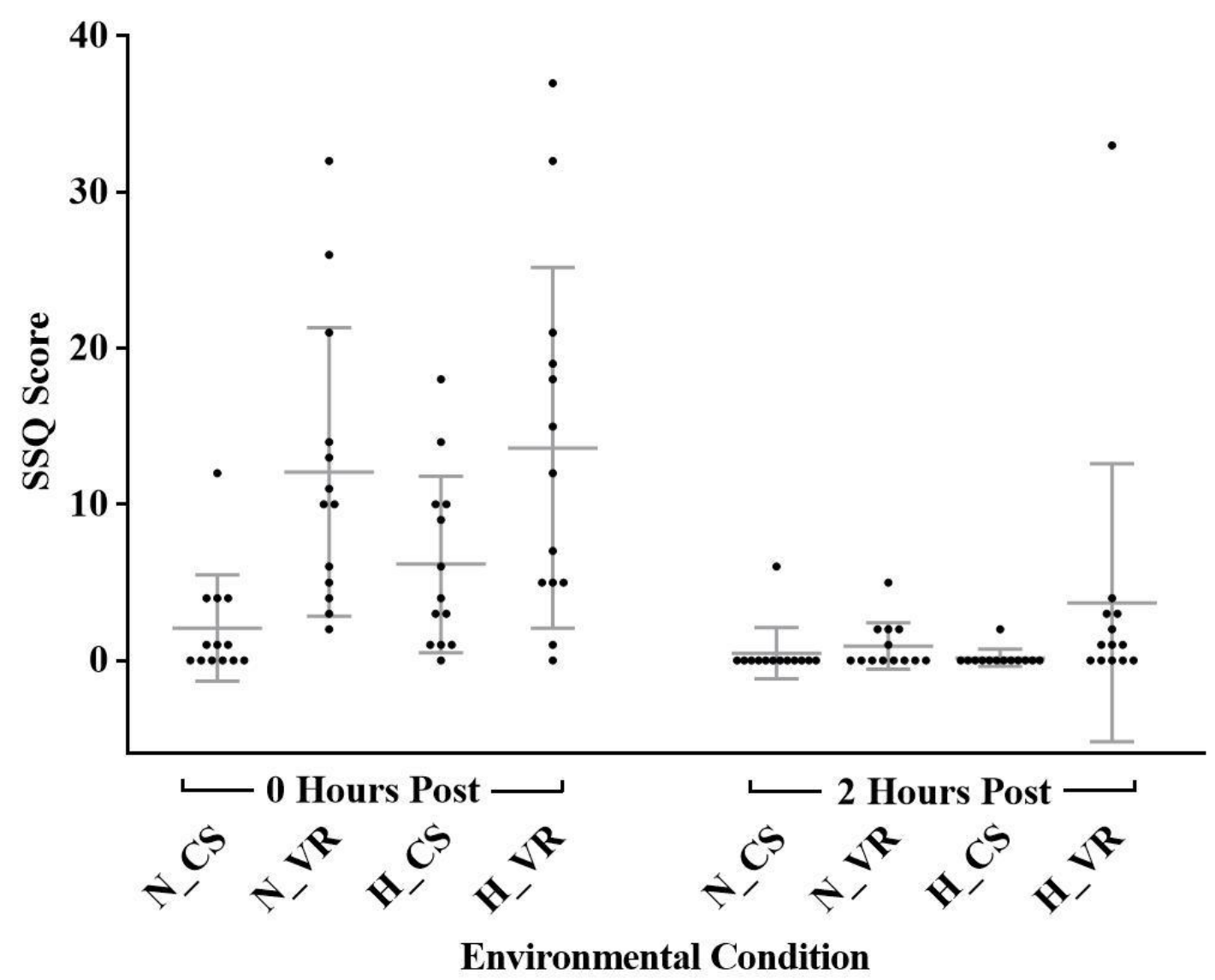

Figure 3: Impact of virtual reality use and air temperature on perceptions of visually induced motion sickness assessed via a Simulator Sickness Questionnaire.

Note: Data are mean $\pm S D$, with individual data points presented; $n=13$. Experimental conditions; N_CS, $22^{\circ} \mathrm{C}$ Ta with computer screen; N_VR, $22^{\circ} \mathrm{C}$ Ta with virtual reality headset; H_CS, $35^{\circ} \mathrm{C}$ Ta with computer screen; H_VR, $35^{\circ} \mathrm{C}$ Ta with virtual reality 


\subsection{Physiological Parameters}

243 No differences $(\mathrm{p}>0.2)$ were observed in physiological variables at baseline across

244 experimental conditions upon immediate entry to the room. Independently, VR significantly 245 increased local sweat rate (CS trials, $45.6[36.2-54.9] \mathrm{g} / \mathrm{m}^{2} / \mathrm{hr}$ vs. VR trials, 59.9 [46.6$\left.24673.2] \mathrm{g} / \mathrm{m}^{2} / \mathrm{hr} ; \mathrm{p}=0.001\right)$ and reduced cutaneous vascular conductance (CS trials, 0.34 [0.25 $2470.44]$ au vs. VR trials, 0.26 [0.18 - 0.34] au; $\mathrm{p}=0.02)$. No independent main effect of VR was 248 seen on any other physiological variables $(p>0.6)$. Air temperature significantly increased 249 mean skin temperature ( $N$ trials, $32.1[31.6-32.7]^{\circ} \mathrm{C}$ vs. $H$ trials, $34.7[34.4-35.1]^{\circ} \mathrm{C}$; $250 \mathrm{p}<0.001)$, heart rate $(71.3[68.0-74.6]$ bpm vs. $75.5[72.3-78.6]$ bpm; $p<0.05)$, cutaneous 251 vascular conductance $(0.25[0.17-0.33]$ au vs. $0.36[0.25-0.46]$ au; $p<0.05)$ and local sweat 252 rate $\left(37.4[31.2-43.7] \mathrm{g} / \mathrm{m}^{2} / \mathrm{hr}\right.$ vs. $\left.68.0[50.8-85.2] \mathrm{g} / \mathrm{m}^{2} / \mathrm{hr} ; \mathrm{p}<0.001\right)$. No independent 253 main effects of air temperature were observed in mean arterial pressure or rectal temperature 254 ( $p>0.1$ ). Finally, no interactions were observed between air temperature and VR across any of 255 the measured variables ( $\mathrm{p}>0.1)$ (Table 1). However, as seen in Table 1, a noticeable 256 difference in the magnitude of change between H_CS and H_VR, compared to N_CS and 257 N_VR was observed for sweat rate data (H_VR - H_CS $\Delta, 18.2$ vs. N_VR - N_CS $\Delta, 10.5)$. 258 The was also observed for CVC data (H_VR - H_CS $\Delta, 11.6$ vs. N_VR - N_CS $\Delta, 2.5)$. Peak 259 values for all physiological variables reflect the patterns seen in mean values. Taken together, 260 the data supports a trend for synergism when combining heat and VR on skin blood flow and 261 local sweating responses. 
Table 1: Impact of virtual reality use and air temperature on assessed physiological parameters.

\begin{tabular}{lccccc}
\hline & N_CS & N_VR & H_CS & H_VR & Effects \\
\hline Heart Rate $(\mathbf{b p m})$ & $72 \pm 7$ & $71 \pm 8$ & $74 \pm 7$ & $77 \pm 6$ & $T_{a}$ \\
Systolic Blood Pressure $(\mathbf{m m} \mathbf{H g})$ & $121 \pm 12$ & $121 \pm 13$ & $120 \pm 12$ & $120 \pm 10$ & \\
Diastolic Blood Pressure $(\mathbf{m m} \mathbf{H g})$ & $75 \pm 6$ & $73 \pm 7$ & $73 \pm 6$ & $74 \pm 7$ & \\
Skin Temperature $\left({ }^{\circ} \mathbf{C}\right)$ & $31.9 \pm 1.0$ & $32.3 \pm 1.1$ & $34.7 \pm 0.7$ & $34.8 \pm 0.5$ & $T_{a}$ \\
Rectal Temperature $\left({ }^{\circ} \mathbf{C}\right)$ & $37.1 \pm 0.2$ & $37.2 \pm 0.2$ & $37.2 \pm 0.3$ & $37.0 \pm 0.4$ & \\
Local Sweat Rate $\left(\mathbf{g} / \mathbf{m}^{\mathbf{2}} / \mathbf{h r}\right)$ & $32.2 \pm 9.8$ & $42.7 \pm 14.8$ & $58.9 \pm 24.0$ & $77.1 \pm 30.6$ & $T_{a}, V R$ \\
Cutaneous Vascular $\mathbf{C o n d u c t a n c e}$ & $23.5 \pm 13.8$ & $21.0 \pm 12.9$ & $37.1 \pm 21.4$ & $25.5 \pm 11.9$ & $T_{a}, V R$ \\
\hline
\end{tabular}

Note: Data are mean \pm SD. Main effects determined via paired sampled T-test for air temperature $\left(T_{a}\right)$ and Visual Stimulus $(V R)$, and repeated measures ANOVA for interaction $\left(T_{a} * V R\right) ; n=13$. Experimental conditions; N_CS, $22^{\circ} \mathrm{C} \mathrm{T}_{\mathrm{a}}$ with computer screen; N_VR, $22^{\circ} \mathrm{C} \mathrm{T}_{\mathrm{a}}$ with virtual reality headset; $\mathrm{H} \_\mathrm{CS}, 35^{\circ} \mathrm{C} \mathrm{T}_{\mathrm{a}}$ with computer screen; H_VR, $35^{\circ} \mathrm{C} \mathrm{T}_{\mathrm{a}}$ with virtual reality headset.

\subsection{DISCUSSION}

265 This study aimed to assess the combined and differential impact of a hot air temperature and

266 virtual reality on motion sickness severity and the associated thermoeffectors (sweating,

267 vasomotor activity). In accordance with the aim, three hypotheses are discussed:

\subsection{Hypothesis 1 - Independently of $T_{a}, V R$ use would elicit motion sickness}

270 In agreement with hypothesis 1, the results of the present study highlight a main effect of VR,

271 indicating a significant increase in perceptions of VIMS with VR use relative to a 2D

272 computer screen control. This finding supports previous research, including Akiduki et al. [3]

273 and Ohyama et al. [4], who also demonstrated evidence for VIMS with VR; a consideration

274 for both providers and users of VR. It appears that symptoms of VIMS may present

275 themselves with as little as 10-20 mins of provocative VR exposure, aligning with previous

276 data [26]. Importantly, this finding shows that the VR stimulus utilised within the current

277 study was sufficient to elicit VIMS in the present participant cohort. 
Findings across physiological parameters provide additional support for hypothesis 1 . An increase in sweat rate was seen with VR use, highlighting the presence of 'cold-sweating', a well-documented symptom of motions sickness. Indeed, a correlation of sweat rate with nausea and VIMS has previously been determined at $r=0.67, \mathrm{p}<0.01$ (Nalivaiko, Rudd and So, 2014). Numerous other studies have documented increased sweating response during motion sickness [27-30]. Interestingly, the current investigation reported a significant reduction in CVC with VR use compared to a CS control, perhaps resulting from increased sympathetic nervous drive during sickness. Previous literature addressing the link between motion sickness and CVC remains inconclusive [20], with some studies highlighting a reduction in CVC during exposure to a provocative stimulus [31] and others highlighting an increase in CVC $[19,32]$. No changes were observed in rectal temperature, likely due to the short nature of each experimental trial.

\subsection{Hypothesis 2 - Independently of VR, hot conditions would not elicit motion sickness}

Physiological parameters responded in a manner expected relative to increases in air temperature [33]. Results showed significant increases in the sweat rate, skin blood flow, skin temperature and heart rate in hot conditions relative to the neutral control. No change was seen in rectal temperature across trials due the short exposure time used in the present study.

Alternatively, mixed findings were observed in accordance with hypothesis 2 ; in agreement, no main effect was observed for air temperature on FMS ratings, however, a significant main effect for air temperature was seen in SSQ scores. Though there appears to be no clear physiological rationale for which hot air conditions alone may directly elicit motion sickness, one must consider whether general feelings of discomfort that individuals experienced as a 
reporting. Indeed, as part of the 16-point SSQ to assess VIMS, individuals were asked to rate their perceptions of 'general discomfort', 'sweating' and 'fatigue'; symptoms which are elevated under hot conditions. While all participants were asked to only report on motion sickness related symptoms, it is not always possible for participants to delineate the effects of

308 thermoregulatory sweating and discomfort, against that of VIMS induced symptoms. Finally, 309 attention paid to psychological constructs may help further to explain increased SSQ ratings with heat alone in the current study. Conceivably, anxiety would increase with the discomfort experienced during hot trials. Indeed, considering the symptoms of anxiety, which also include increased stomach upset, dizziness and heart rate, one could consider anxiety as another rationale as to why SSQ reporting increased in hot conditions alongside the spill over

314 of thermoregulatory responses [34]. Whilst the links between trait anxiety and motion sickness have been previous explored $[35,36]$, little research has investigated whether state anxiety in response to heat stress may also add to the onset of motion sickness.

\subsection{Hypothesis 3 - Combined effect of heat and VR on motion sickness}

319 The findings of the present study do not support hypothesis 3, providing no clear evidence for a synergistic interaction between increased air temperature and VR. Notwithstanding, a noticeable difference in the magnitude of change between H_CS and H_VR trials, compared to N_CS and N_VR trials for FMS and SSQ, indicates potential signs of synergism in some, but not all participants. Indeed, a closer look at individual FMS and SSQ data shows partial

324 evidence for responders and non-responders (Fig $2 \& 3$ ). This is supported by the findings of many large scale studies which report disparity in the motion sickness susceptibility across

326 individuals exposed to an identical provocative stimulus, either artificial or true motion $327[10,37]$. Indeed, the presence of non-responders in the study cohort limits the power available 328 for the interaction statistic, thereby potentially masking any synergistic effects of heat on VR 
induced motion sickness. Note, due the limited sample size within the current study, it was not possible to partition the data into groups to explore this further. A repeat of the current investigation, utilizing only individuals who are known to 'respond' to motion sickness would 332 provide an intriguing area of investigation; however, this may also reduce the generalisability 333 of the results to a wider population of users.

335 It is also plausible that modifications made to the magnitude and type of visual VR stimulus 336 may in turn increase the magnitude and consistency of any potential interaction between VR 337 induced vection and heat. Though it was conjectured that a fast-moving motor sport video 338 sequence via VR would elicit vection and subsequently motion sickness, in addition to the 339 positive findings discussed for hypothesis 1 , future investigations may wish to trial a video 340 sequence with a wider array of planes of motion; e.g. motion experienced on a rollercoaster or 341 simulated human movement through a range of obstacles. In support, Bonato and colleagues $342[23,38]$ found that some VR stimuli are effective in evoking VIMS, while others are less so.

343 Note, the extent of vection directly experienced by participants was not assessed in the current 344 investigation, thus should be included in future research. Investigation into the combined 345 stress of heat and true motion, in place of VR, for direct comparison to the current study, also 346 provides an intriguing area for future exploration.

348 Physiological parameters do not provide unequivocal evidence for synergism between $\mathrm{T}_{\mathrm{a}}$ and 349 VR. Yet, in parallel with the subjective motion sickness findings, analysis of sweat rate and 350 cutaneous vascular conductance show potential trends towards a synergistic interaction 351 between heat exposure and VR use. As seen in Table 1, a difference in the magnitude of 352 change between H_CS vs. H_VR trials, compared with N_CS vs. N_VR trials, shows 353 evidence for increased sudomotor activity when VR and heat are combined. Interestingly, 
vasomotor activity showed conflict between the vasoconstriction induced by VR and vasodilatation induced by heat. In view of the toxic hypothesis, such findings are intriguing and worth further investigation; yet due to the large inter-individual variation across the current data set, an unequivocal conclusion is not possible.

\subsection{Conclusion}

360 This investigation assessed the individual and combined impact of VR use and a hot air 361 temperature on motion sickness severity. Independently, VR evoked a significant increase in 362 self-reported motion sickness. Nonetheless, the data herein does not provide unequivocal 363 evidence of a clear synergistic interaction between VR and $\mathrm{T}_{\mathrm{a}}$. While definitive evidence for a 364 synergistic interaction was not obtained, potential trends were identified that warrant further investigation. Considerable variation was seen in the inter-individual resistance to motion sickness, conceivably limiting the statistical power available for a significant interaction between stressors.

\section{REFERENCES}

370 [1] E.. Regan, K.. Price, The frequency of occurrence and severity of side-effects of immersion virtual reality., Aviat. Space. Environ. Med. 65 (1994). http://psycnet.apa.org/record/1994-41790-001 (accessed March 21, 2018).

[2] S. Sharples, S. Cobb, A. Moody, J.R. Wilson, Virtual reality induced symptoms and effects (VRISE): Comparison of head mounted display (HMD), desktop and projection display systems, Displays. 29 (2008) 58-69. doi:10.1016/j.displa.2007.09.005. vestibular conflict induced by virtual reality in humans, Neurosci. Lett. 340 (2003) 197200. doi:10.1016/S0304-3940(03)00098-3. 
[4] S. Ohyama, S. Nishiike, H. Watanabe, K. Matsuoka, H. Akizuki, N. Takeda, T. Harada, Autonomic responses during motion sickness induced by virtual reality, Auris Nasus Larynx. 34 (2007) 303-306. doi:10.1016/j.anl.2007.01.002.

[5] B.E. Riecke, J. Schulte-Pelkum, M.N. Avraamides, M. Von Der Heyde, H.H. Bülthoff, Cognitive factors can influence self-motion perception (vection) in virtual reality, ACM Trans. Appl. Percept. 3 (2006) 194-216. doi:10.1145/1166087.1166091.

[6] B.E. Riecke, J. Schulte-Pelkum, F. Caniard, H.H. Bulthoff, Towards lean and elegant self-motion simulation in virtual reality, IEEE Proceedings. VR 2005. Virtual Reality, 2005. 2005 (2005) 131-138. doi:10.1109/VR.2005.1492765.

[7] B. Keshavarz, B.E. Riecke, L.J. Hettinger, J.L. Campos, Vection and visually induced motion sickness: How are they related?, Front. Psychol. 6 (2015) 1-11. doi:10.3389/fpsyg.2015.00472.

[8] J. Munafo, M. Diedrick, T.A. Stoffregen, The virtual reality head - mounted display Oculus Rift induces motion sickness and is sexist in its effects, Exp. Brain Res. 235 (2017) 889-901. doi:10.1007/s00221-016-4846-7.

[9] O.X. Kuiper, J.E. Bos, C. Diels, Vection does not necessitate visually induced motion sickness, Displays. 58 (2019) 82-87. doi:10.1016/j.displa.2018.10.001.

[10] A.G. Solimini, Are There Side Effects to Watching 3D Movies? A Prospective Crossover Observational Study on Visually Induced Motion Sickness, PLoS One. 8 (2013). doi:10.1371/journal.pone.0056160.

[11] K.. Money, Motion Sickness, Physiol. Rev. 50 (1970).

[12] R.S. Kennedy, J. Drexler, R.C. Kennedy, Research in visually induced motion sickness, Appl. Ergon. 41 (2010) 494-503. doi:10.1016/j.apergo.2009.11.006.

[13] A.M. Gavgani, F.R. Walker, D.M. Hodgson, E. Nalivaiko, A comparative study of cybersickness during exposure to virtual reality and " classic" motion sickness : are they 
404

405

406

407

408

409

410

411

412

413

414

415

416

417

418

419

420

421

422

423

424

425

426

427

428

different ?, J. Appl. Physiol. (2018) 1670-1680. doi:10.1152/japplphysiol.00338.2018.

[14] J.R. Lackner, Motion sickness: More than nausea and vomiting, Exp. Brain Res. 232 (2014) 2493-2510. doi:10.1007/s00221-014-4008-8.

[15] J. Reason, J. Brand, Motion sickness., 1975. http://psycnet.apa.org/psycinfo/197612574-000 (accessed January 15, 2018).

[16] G.E. Riccio, T.A. Stoffregen, An ecological Theory of Motion Sickness and Postural Instability An Ecological Theory of Motion Sickness and Postural Instability, Ecol. Psychol. 3 (1991) 195-240. doi:10.1207/s15326969eco0303.

[17] M. Treisman, Motion sickness: an evolutionary hypothesis, Science (80-. ). 197 (1977) 493-495. doi:10.1126/science.301659.

[18] I.B. Mekjavic, M.J. Tipton, M. Gennser, O. Eiken, Motion sickness potentiates core cooling during immersion in humans, J. Physiol. 535 (2001) 619-623. doi:10.1111/j.1469-7793.2001.00619.x.

[19] G. Nobel, O. Eiken, A. Tribukait, R. Kölegård, I.B. Mekjavic, Motion sickness increases the risk of accidental hypothermia, Eur. J. Appl. Physiol. 98 (2006) 48-55. doi:10.1007/s00421-006-0217-6.

[20] E. Nalivaiko, J.A. Rudd, R.H. So, Motion sickness, nausea and thermoregulation: The "toxic" hypothesis, $\quad$ Temperature. $\quad 1 \quad$ (2014) 164-171. doi: $10.4161 / 23328940.2014 .982047$.

[21] A. Lloyd, G. Havenith, Interactions in human performance: An individual and combined stressors approach, $\quad$ Temperature. $\quad 3 \quad$ (2016) 514-517. doi:10.1080/23328940.2016.1189991.

[22] C.H. Chang, W.W. Pan, F.C. Chen, T.A. Stoffregen, Console video games, postural activity, and motion sickness during passive restraint, Exp. Brain Res. 229 (2013) 235242. doi:10.1007/s00221-013-3609-y. 
[23] F. Bonato, A. Bubka, S. Palmisano, D. Phillip, G. Moreno, Vection Change Exacerbates Simulator Sickness in Virtual Environments, Presence Teleoperators Virtual Environ. 17 (2008) 283-292. doi:10.1162/pres.17.3.283.

[24] S. Bouchard, G. Robillard, P. Renaud, Revising the factor structure of the simulator sickness quesionnaire, Annu. Rev. Cyber Ther. Telemed. 5 (2007) 117-122.

[25] B. Keshavarz, H. Hecht, Validating an efficient method to quantify motion sickness, Hum. Factors. 53 (2011) 415-426. doi:10.1177/0018720811403736.

[26] P.A. Howarth, S.G. Hodder, Characteristics of habituation to motion in a virtual environment, Displays. 29 (2008) 117-123. doi:10.1016/j.displa.2007.09.009.

[27] H. Wan, S. Hu, J. Wang, Correlation of phasic and tonic skin-conductance responses with severity, Percept. Mot. Skills. (2003) 1051-1057.

[28] N. Himi, T. Koga, E. Nakamura, M. Kobashi, M. Yamane, K. Tsujioka, Differences in autonomic responses between subjects with and without nausea while watching an irregularly oscillating video, Auton. Neurosci. Basic Clin. 116 (2004) 46-53. doi:10.1016/j.autneu.2004.08.008.

[29] S. Hu, W.F. Grant, R.M. Stern, K.L. Koch, Motion sickness severity and physiological correlates during repeated exposures to a rotating optokinetic drum., Aviat. Space. Environ. Med. 62 (1991) 308-14. http://www.ncbi.nlm.nih.gov/pubmed/2031631 (accessed May 8, 2018).

[30] S. Hu, K.A. McChesney, K.A. Player, A.M. Bahl, J.B. Buchanan, J.E. Scozzafava, Systematic investigation of physiological correlates of motion sickness induced by viewing an optokinetic rotating drum., Aviat. Space. Environ. Med. 70 (1999) 759-65. http://www.ncbi.nlm.nih.gov/pubmed/10447048 (accessed May 8, 2018).

[31] G.H. Crampton, Studies of Motion Sickness: XVII. Physiological Changes Accompanying Sickness in Man, J. Appl. Physiol. 7 (1955) 501-507. 
doi:10.1152/jappl.1955.7.5.501.

455

456

457

458

459

460

461

462

463

464

465

466

467

468

469

470

471

472

473

474

475

476

[32] B. Cheung, K. Hofer, Coriolis-induced cutaneous blood flow increase in the forearm and calf, Brain Res. Bull. 54 (2001) 609-618. doi:10.1016/S0361-9230(01)00463-4.

[33] K. Parsons, Human Thermal Environments: The Effects of Hot, Moderate, and Cold Environments on Human Health, Comfort, and Performance, 3rd ed., CRC Press, 2014. doi:10.1201/b16750.

[34] S. Bouchard, G. Robillard, P. Renaud, F. Bernier, Exploring new dimensions in the assessment of virtual reality induced side effects, J. Comput. Inf. Technol. 1 (2011) 2032.

[35] A.C. Paillard, G. Quarck, F. Paolino, P. Denise, M. Paolino, J.F. Golding, V. GhulyanBedikian, Motion sickness susceptibility in healthy subjects and vestibular patients: Effects of gender, age and trait-anxiety, J. Vestib. Res. Equilib. Orientat. 23 (2013) 203210. doi:10.3233/VES-130501.

[36] E. Faugloire, C.T. Bonnet, M.A. Riley, B.G. Bardy, T.A. Stoffregen, Motion sickness, body movement, and claustrophobia during passive restraint, Exp. Brain Res. 177 (2007) 520-532. doi:10.1007/s00221-006-0700-7.

[37] S.C. Stevens, M.G. Parsons, Effects of motion at sea on crew performance: A survey, Mar. Technol. Sname News. 39 (2002) 29-47.

[38] F. Bonato, A. Bubka, S. Palmisano, Combined Pitch and Roll and Cybersickness in a Virtual Environment, Aviat. Space. Environ. Med. 80 (2009) 941-945. doi:10.3357/ASEM.2394.2009. 


\section{AUTHOR BIO}

478

479

Josh T. Arnold (MRes) - Josh Arnold is a PhD researcher in the Environmental Ergonomics Research Centre at Loughborough University. His research seeks to investigate the individual and combined impact of multifactorial extreme environments on human function and capacity. Prior to this, Josh held a research position at Southampton Solent University, publishing across numerous topics including translational physiology and public health. In 2012 Josh studied for a masters by research degree at Bangor University focusing on the physiology of sport and exercise.

Kate O'Keeffe (MSc) - Kate O'Keeffe is a current $\mathrm{PhD}$ researcher at the Environmental Ergonomics Research Centre, Loughborough University, UK. Her research surrounds cognitive and physical performance in extreme environments. She completed a double master's degree in sports and exercise psychology and diagnostics and intervention studies from the University of Jyväskylä, Finland and Leipzig University, Germany respectively in 2016. She is also a qualified and experienced physical education and mathematics teacher.

Chloe McDaniel (MSc) - Chloe is an ergonomist working as an occupational health and safety consultant for Human Applications based in Loughborough, England. Prior to working in health and safety, Chloe received her MSc in Ergonomics and Human Factors (2017) from Loughborough University. She completed her master's thesis in the Environmental Ergonomics Research Centre, exploring the effects of environmental temperatures on motion sickness when using virtual reality.

\section{Simon Hodder (PhD) - Dr Simon Hodder is a Senior Lecturer in the Environmental} Ergonomics Research Centre in the Loughborough Design School. He has over 20 years' 
experience working in the field of environmental ergonomics: thermal, vision and virtual

503 reality. He is a scientific editor for Applied Ergonomics and is Chairman of

504 ISO/TC159/Scientific Committee 5 (Ergonomics of the Physical Environment).

506 Alex Lloyd (PhD) - Dr Alex Lloyd is a researcher and full time academic in Environmental

507 Ergonomics and Human Physiology at Loughborough University. After working for the

508 Royal Air Force Centre of Aviation Medicine, Dr Lloyd attained his PhD in Neuromuscular

509 Physiology from Loughborough University. Dr Lloyd has since published widely across the

510 fields of Integrative Physiology, Temperature Regulation and Environmental Ergonomics. His

511 current research is centred on the impact of multiple environmental stressors on human

512 physical and cognitive performance. Dr Lloyd is an Associate Editor at the journal

513 Ergonomics.

514

515 
